\title{
Model Experiment for Acceleration of Lime Dissolution into Slag under Ultrasound Irradiation Conditions
}

\author{
Keiji OKUMURA* \\ Graduate School of Engineering, Nagoya Institute of Technology, Gokiso-cho, Showa-ku, Nagoya, $466-8555$ Japan.
}

(Received on April 17, 2017; accepted on August 8, 2017)

\begin{abstract}
In the metal manufacturing process, it is considered that ultrasound technology can be applied in the promotion of solid dissolution such as lime dissolution to molten slag and solid metal addition to molten alloy. Generally, in the high-temperature reaction process, mass transfer has a large effect on the rate of reaction in isothermal conditions. In order to facilitate mass transfer, it is necessary to strengthen the agitation of the bath. However, it is impossible to avoid the erosion of the stirrer and container. Enhancement of stirring promotes mass transfer. This corresponds to thinning of the boundary layer thickness in the boundary layer theory. To further accelerate mass transfer, a new method of thinning the boundary layer of the solid surface without using mechanical stirring is needed.

In this study, the effect of ultrasound on the dissolution phenomena of solid in liquid was investigated by using a sucrose-water system. Applying ultrasound to a liquid induces ultrasonic radiation pressure, acoustic streaming and various nonlinear phenomena of cavitation. Especially, when the cavitation bubble collapses, a micro jet is generated and a strong flow occurs locally. It was thought that this micro jet collided with the solid sample and the surface became uneven. If the cavitation phenomena occur at the solid surface, it is possible to disturb the boundary layer and increase the mass transfer rate by thinning the boundary layer. It was found that the cavitation in the vicinity of the solid surface at the low ultrasound frequency caused a disturbance of the boundary layer of the solid surface and promoted dissolution rate of the solid.
\end{abstract}

KEY WORDS: ultrasound; solid dissolution rate; cavitation; mass transfer.

\section{Introduction}

Currently, ultrasound technology has been used in a wide range of fields such as engineering, medicine and materials science. The dynamic applications in ultrasound are still expanding and have the potential to creating many applications of materials science. ${ }^{1)}$

Irradiation of ultrasound to molten metal can result in the promotion of degassing, ${ }^{2,3)}$ aggregation or removal of inclusions ${ }^{4}{ }^{4}$ dissolution of solid metal, grain refinement ${ }^{5)}$ and prevention of segregation of solidification structure. In particular, ultrasound can be utilized for a long time to control the solidification structure in the casting field, and many studies have been carried out. ${ }^{6}$

In the metal manufacturing process, it is considered that ultrasound technology can be applied in the promotion of solid dissolution such as lime dissolution to molten slag ${ }^{7}$ and solid metal addition to molten alloy. Generally, in the high-temperature reaction process, mass transfer has a large effect on the rate of reaction in isothermal conditions. In order to facilitate mass transfer, it is necessary to strengthen the agitation of the bath. However, it is impossible to avoid the erosion of the stirrer and container. To further facilitate

* Corresponding author: E-mail: okumura@nitech.ac.jp

DOI: http://dx.doi.org/10.2355/isijinternational.ISIJINT-2017-208 mass transfer, a new method of thinning the boundary layer of the solid surface without using mechanical stirring is needed.

Recently, Yamakado et al. ${ }^{8)}$ investigated the effect of the ultrasound on the boundary layer formed in an aqueous solution by anodic reaction of a copper electrode. They pointed out that the ultrasound irradiation reduces the concentration boundary layer thickness. Said et al. ${ }^{9)}$ investigated the effect of sonication on the dissolution of calcium from steel slag by $1 \mathrm{M}$ aqueous solution of ammonium chloride. They compared the experimental results from similar tests with mechanical agitation.

Applying ultrasound to a liquid induces the ultrasonic radiation pressure, acoustic streaming ${ }^{10)}$ and various nonlinear phenomena of cavitation. ${ }^{11)}$ If the cavitation is activated in the vicinity of the solid surface, the mass transfer is promoted by micro-jet when the cavitation bubble collapses. ${ }^{12,13)}$ Therefore, dissolution rate is promoted by thinning or destroying the concentration boundary layer formed on the surface of the solid. ${ }^{14-16)}$

In general, a Langevin-type ultrasound transducer is used to irradiate ultrasound into a liquid, but the performance of the ultrasound transducer deteriorates in a high temperature system. In this study, we investigated the influence of ultrasound on the dissolution rate of solid by using a low temperature, sucrose-water, model system. 


\section{Experimental}

Figure 1 shows a schematic diagram of the experimental apparatus. An alumina vessel, O.D. $36 \mathrm{~mm}$, I.D. $30 \mathrm{~mm}$, $25 \mathrm{~mm}$ height or an aluminum vessel, O.D. $15 \mathrm{~mm}$, I.D. $14 \mathrm{~mm}, 25 \mathrm{~mm}$ height is used for the experiment. $10 \mathrm{~cm}^{3}$ or $3 \mathrm{~cm}^{3}$ of distilled water is poured into the alumina container or the aluminum container, respectively. Since the wall surface of the aluminum vessel is thin, the wall surface on which the sound wave hits vibrates. Therefore, the reflected sound is irradiated to all directions in the container again. The cavitation phenomenon in the liquid becomes much active for the aluminum container. Degassed distilled water obtained by vacuum degassing was also used for the experiment. A solid sucrose was pre-heated and melted to mold into a cylindrical shape of O.D. $4 \mathrm{~mm}$ and $30 \mathrm{~mm}$ length. After the solid sample was approximately $20 \mathrm{~mm}$ immersed in water, the ultrasound was applied by the Langevin type ultrasound vibrator installed on the vessel bottom. The ultrasound power was adjusted by amplifying the sine wave signal from the function generator by the high speed power amplifier. The frequency of ultrasound was 28 and $108 \mathrm{kHz}$. After applying ultrasound wave at a predetermined output and time, the sample was taken out and dried. To calculate the sucrose concentration in water, the dissolved amount was measured from the mass difference of the sample before and after the experiment. The sucrose concentration was also measured by using a Brix meter. It was confirmed that the concentration obtained from the sample weight loss was equal to the concentration measured by a Brix meter. For this reason, the concentration obtained from the sample weight loss was used. The surface area of the dissolved sample was calculated from the diameter change of the sample. The diameter of the dissolved sample was measured at three points, the upper part, the central part and the bottom part, and the surface area was obtained assuming a truncated cone shape. We also assumed no irregularities on the surface and a smooth surface. The ultrasound power was measured by

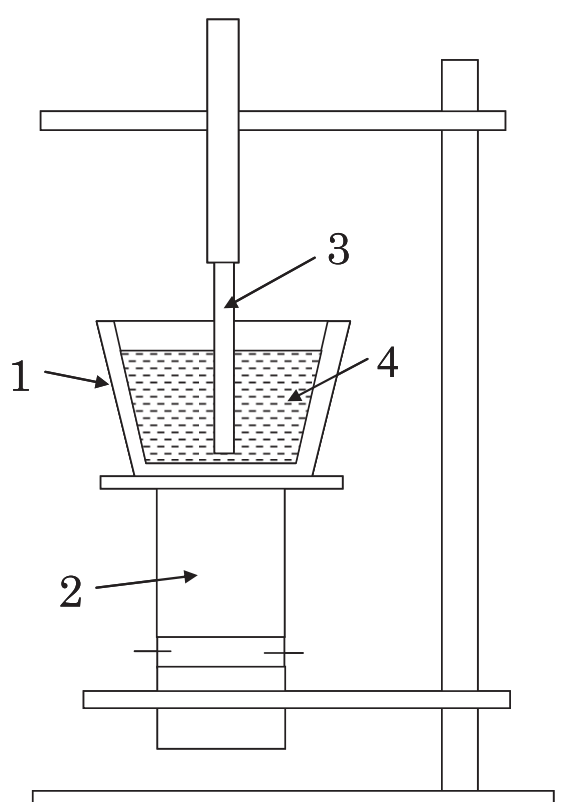

Fig. 1. Experimental apparatus. 1: vessel, 2: ultrasound vibrator, 3: sucrose rod, 4: water. the calorimetry method. ${ }^{17)}$ This method is based on the fact that the ultrasound vibration energy is ultimately converted into heat. The vessel containing water was covered with a heat insulating material and the rising rate of the water temperature was measured while irradiating with ultrasonic waves. The ultrasound power, $P_{W}$, was calculated by the following equation.

$$
P_{W}=\frac{d T}{d t} V C_{P} \rho
$$

where $T(\mathrm{~K})$ is temperature, $t(\mathrm{~s})$ is time, $V\left(\mathrm{~cm}^{3}\right)$ is water volume, $C_{p}\left(\mathrm{~J} \cdot \mathrm{g}^{-1} \cdot \mathrm{K}^{-1}\right)$ is specific heat of water, and $\rho\left(\mathrm{g} / \mathrm{cm}^{3}\right)$ is density of water. Under this experimental condition the temperature rise was up to $5 \mathrm{~K}$. Therefore, the changes in solubility and diffusion coefficient of sucrose in liquid were ignored.

\section{Results and Discussion}

\subsection{Effect of Ultrasound Power on Dissolution Rate}

Figure 2 shows photographs of samples after the experiment using non-degassed water at ultrasonic frequency of $28 \mathrm{kHz}$. These are experimental results using alumina vessels. Figure 2(a) is with no application of ultrasound wave and (b) is for the case of $1.07 \mathrm{~W}$ of ultrasound power. When no ultrasound was applied, the sample surface was smooth and the shape was changed from a cylinder to a circular truncated cone. This is because the descending of high concentration solution along the sample surface delays the dissolution rate of the sample bottom. On the other hand, the sample surface becomes uneven under ultrasound irradiation conditions. The lower part of the dissolved sample was narrowed. The disturbance caused by cavitation phenomena has become active in the sample bottom close to the ultrasound vibrating surface. Therefore, it is considered that the sample surface has been eroded more.

Figure 3 is a sample photo of after experiments with degassed water. Although the ultrasound output of Fig. 3 is smaller than that of Fig. 2(b), it can be seen that the erosion of the sample bottom is intense. The lower end of the sample was dropped after $150 \mathrm{~s}$. There are two types of phenomenon of gaseous cavitation and vaporous cavitation. The gaseous cavitation is dependent on the release of dissolved gases, while the vaporous cavitation is dependent on the vapor pressure of the liquid. The gaseous cavitation bubbles grow relatively large, on the other hand, the vaporous cavitation bubbles are very small. These tiny cavitation bubbles occur locally and very strong flow called micro-jet occurs when they collapse. The micro-jets have eroded the sucrose surface and the dissolution rate of the sample has been promoted. The degassed water is more likely to cause micro jet due to vaporous cavitation than non-degassed water.

Figure 4 shows the relationship between surface area of the sample and time. Linear relationships are obtained in all conditions. The surface area of the sample can be expressed by a linear equation with respect to time in this experimental condition.

$$
S=a t+b
$$

Where $a, b$ are constants.

When the rate controlling step of the sucrose dissolution 

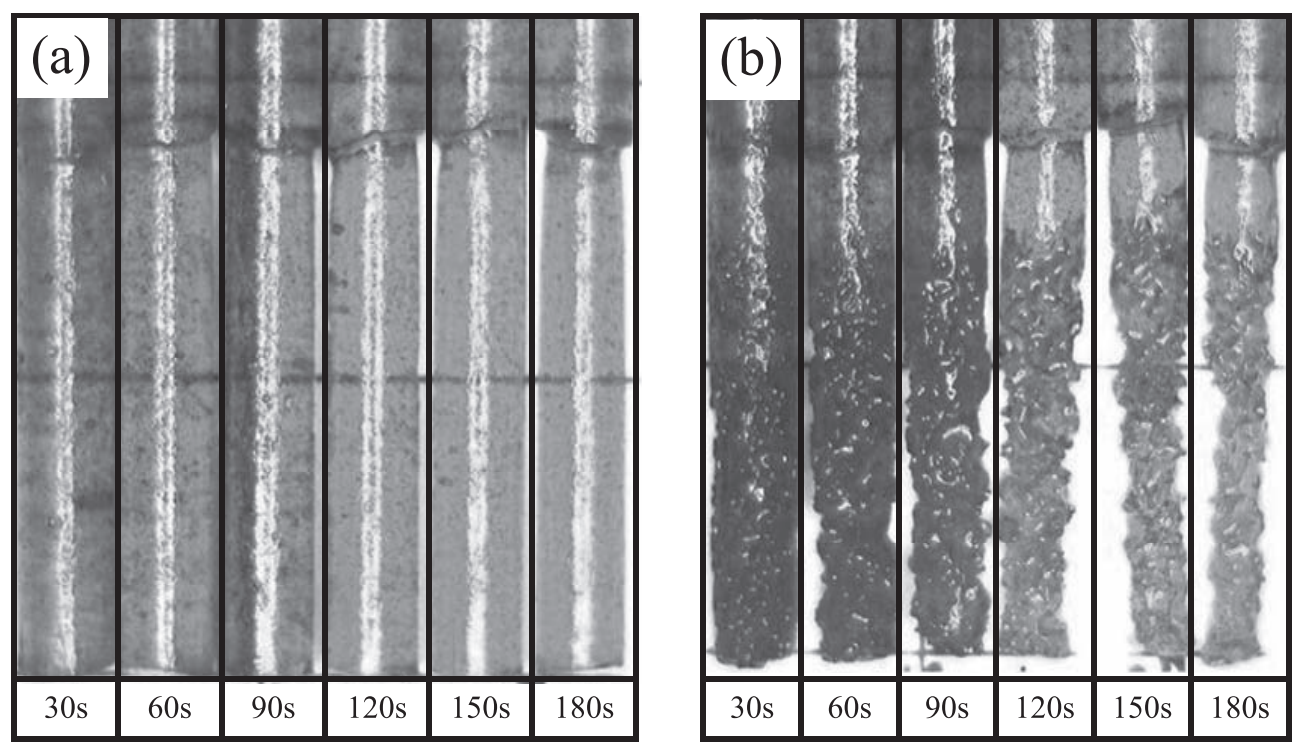

Fig. 2. Change in shape due to dissolution of the sucrose samples by using non-degassed distilled water. (a) without ultrasound (b) with ultrasound ( $28 \mathrm{kHz} 1.07 \mathrm{~W})$.
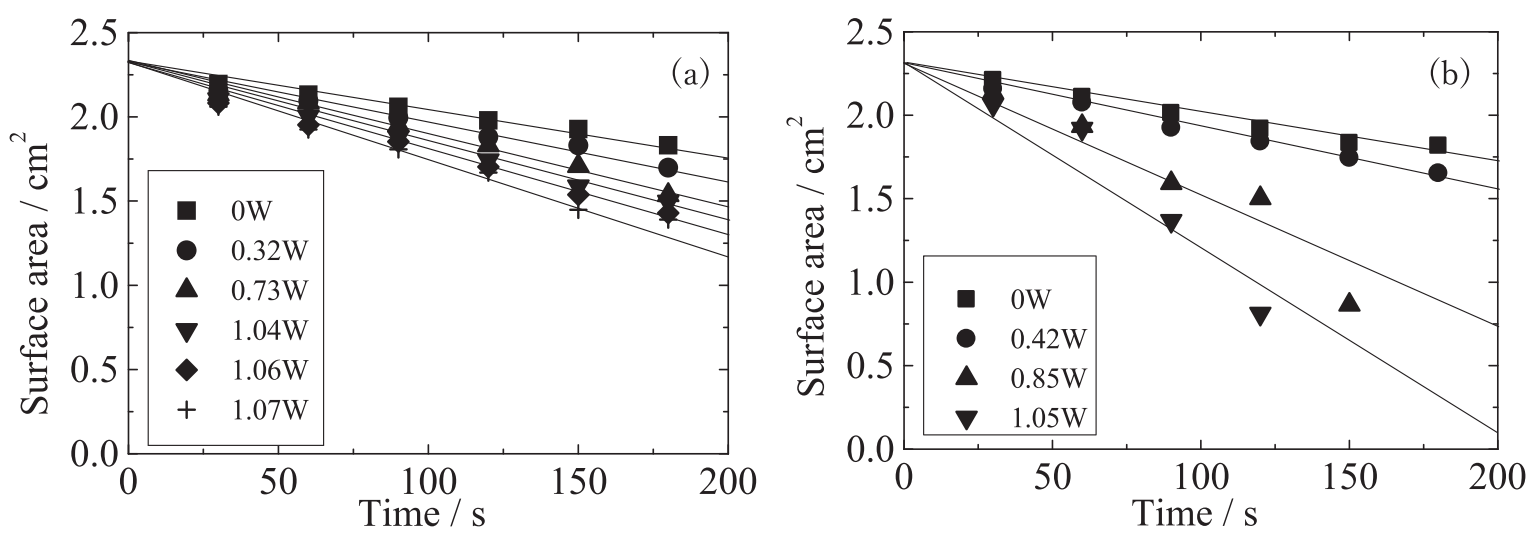

Fig. 4. Comparison of time variation of the surface area of the sample due to differences in the ultrasonic output in the case of $28 \mathrm{kHz}$. (a) non-degassed distilled water (b) degassed distilled water.

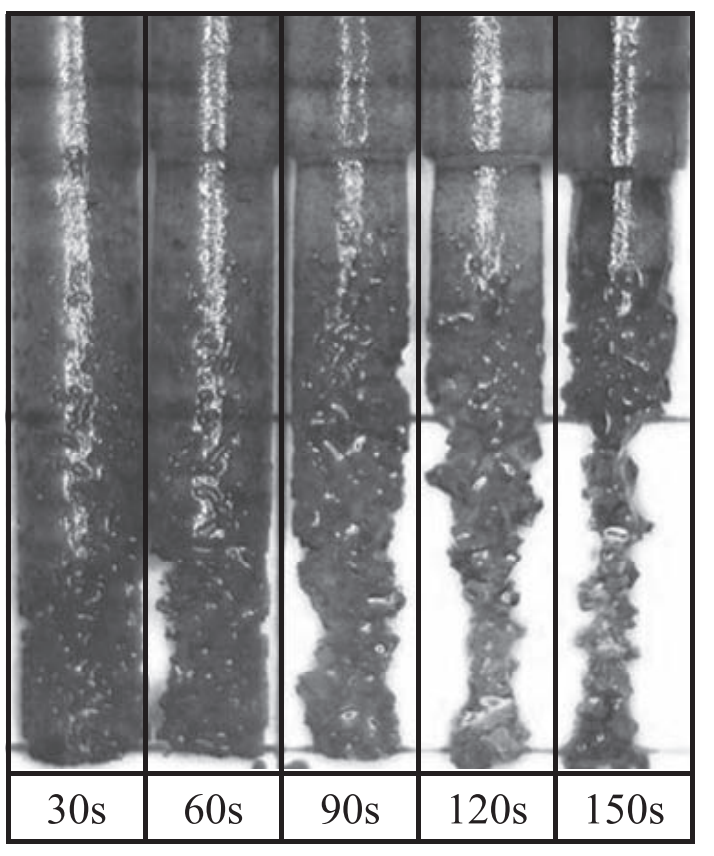

Fig. 3. Change in shape due to dissolution of the sucrose samples by using degassed distilled water under ultrasound irradiation condition $(28 \mathrm{kHz} 0.85 \mathrm{~W})$. is assumed to be mass transfer rate in the liquid, it can be applied as a first order rate equation. The time change in the concentration is expressed as follows.

$$
-\frac{d C}{d t}=\frac{S}{V} k\left(C^{*}-C\right)
$$

Where $k(\mathrm{~cm} / \mathrm{s})$ is mass transfer coefficient, $C^{*}(\operatorname{mass} \%)$ is the saturating concentration. After substituting Eq. (2) into Eq. (3), the following equation can be obtained by integrating the equation.

$$
\ln \frac{C^{*}-C_{0}}{C^{*}-C}=\frac{k}{V}\left(\frac{a}{2} t^{2}+b t\right) .
$$

Where $C_{0}(\operatorname{mass} \%)$ is the initial concentration, in this case $C_{0}=0$.

Figure 5 shows summarized experimental results by using Eq. (4). Linear relationship is obtained in all conditions. Mass transfer coefficient is calculated from the slope of these straight lines.

Figure 6 shows the influence of ultrasound power on the mass transfer coefficient. It was found that mass transfer coefficient increases as the ultrasound power is increased. 

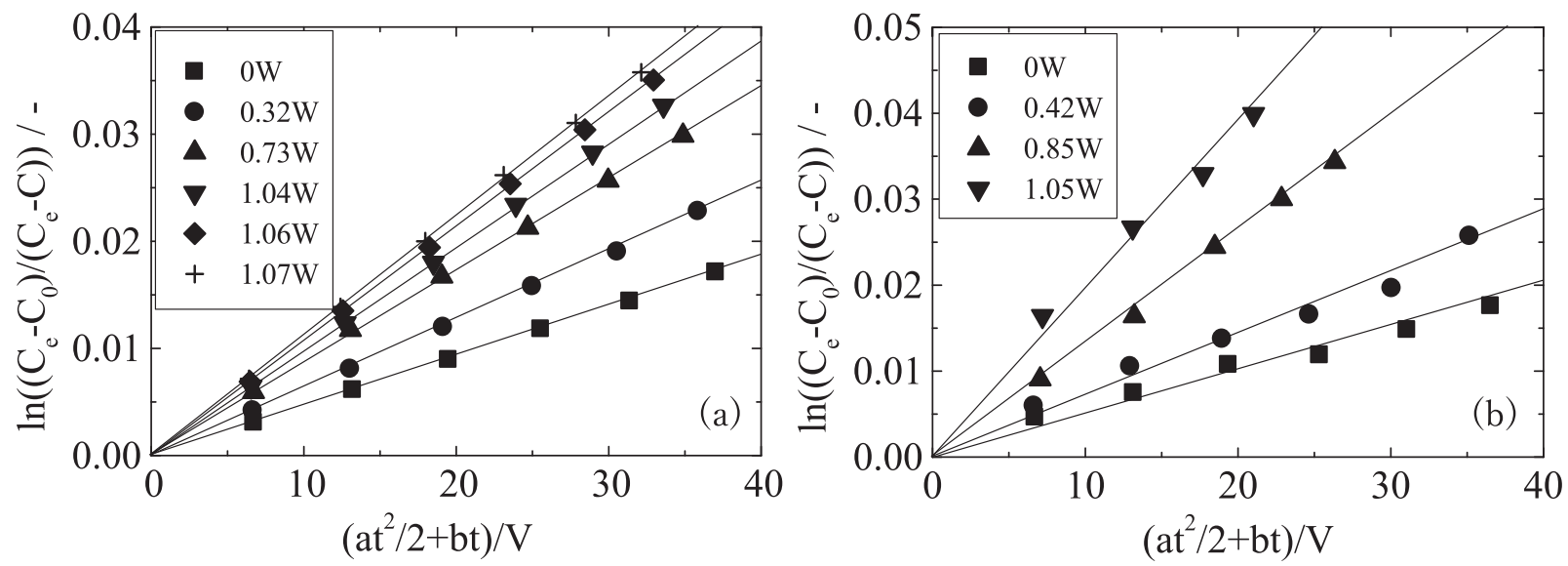

Fig. 5. Analysis by the rate equation of the time-dependent change of sucrose concentration in the case of $28 \mathrm{kHz}$. (a) non-degassed distilled water (b) degassed distilled water.

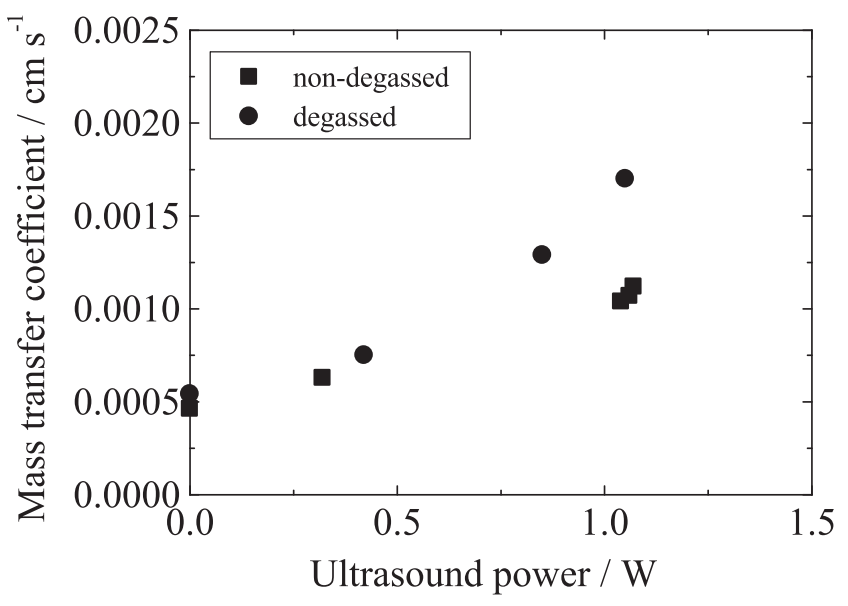

Fig. 6. Influence of ultrasound power on the mass transfer coefficient in the case of $28 \mathrm{kHz}$.

Further, the mass transfer coefficient of the degassed water is greater than that of the non-degassed water. This is because of the active micro-jets formed by the collapse of vaporous cavitation bubbles in the case of degassed water.

The dependence of dissolution kinetics on ultrasound power is usually expressed by the following relation. ${ }^{18,19)}$

$$
E_{W}=\left(1+\alpha P_{W}\right)^{\beta}
$$

Where $E_{W}$ is the ultrasonic enhancement factor, calculated as

$$
E_{W}=\frac{k_{P_{W}}}{k_{0}}
$$

where $k_{P_{W}}(\mathrm{~cm} / \mathrm{s})$ is mass transfer coefficient under ultrasound irradiation conditions, $k_{0}(\mathrm{~cm} / \mathrm{s})$ is mass transfer coefficient when ultrasound wave is not irradiated. In Eq. (5), $\alpha$ and $\beta$ are experimental constants estimated by non-linear regression analysis. Figure 7 shows the relationship between $E_{W}$ and calculated values according to Eq. (5). The vertical axis and the horizontal axis are logarithmic. The fit is sufficiently good to confirm Eq. (5). Finally, the ultrasonic enhancement factor was obtained by the following experimental formula in the case of non-degassed water.

$$
E_{W}=\left(1+1.53 P_{W}\right)^{0.84}
$$

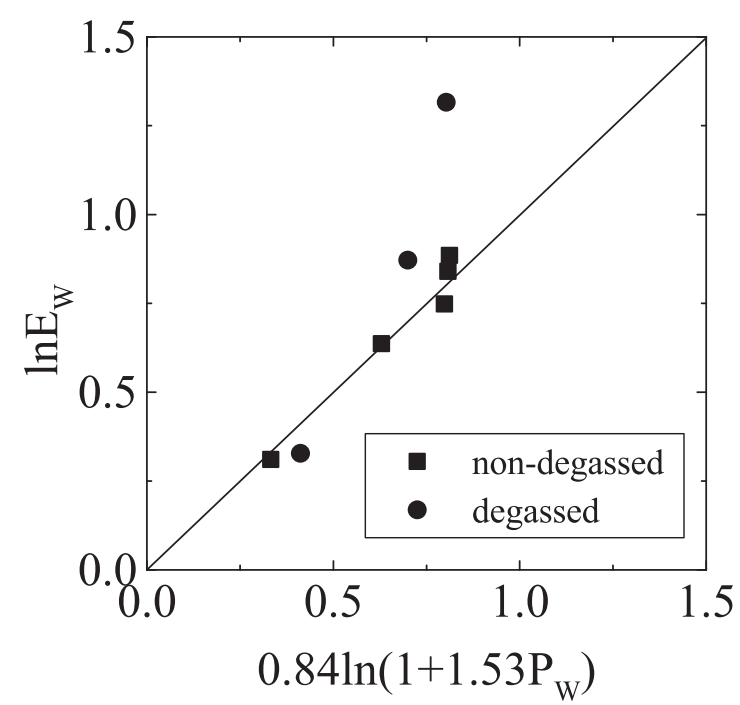

Fig. 7. Determination of the parameters of Eq. (5) showing a relationship between the ultrasonic enhancement factor and ultrasound power in the case of $28 \mathrm{kHz}$.

In the case of degassed water, it is impossible to explain the experimental results by Eq. (7). Since the value of $E_{W}$ obtained from the experiment is larger than the value calculated by Eq. (7), it can be said that the promotion effect of solid dissolution by ultrasound is large when the degassed liquid is used. The reason for this is believed to be that the vaporous cavitation is active in the degassed liquid.

Horst et al. ${ }^{18)}$ reported that $\alpha$ and $\beta$ were 0.23 and 0.5 , respectively. In this study, we obtained larger value of $\alpha$ and $\beta$. These parameters are dependent on the equipment conditions. Therefore, it is necessary to make some experiments by changing the conditions systematically. In the future, we should make clear the physical meaning of the parameters.

\subsection{Effect of Ultrasound Frequency on Dissolution Rate}

Figure 8 shows the relationship between surface area of the sample and time in the case of $108 \mathrm{kHz}$. Linear relationships are obtained in all conditions. Figure 9 shows summarized experimental results by using Eq. (4). Linear relationship is obtained in all conditions. Mass transfer coefficient is calculated from the slope of these straight lines. 

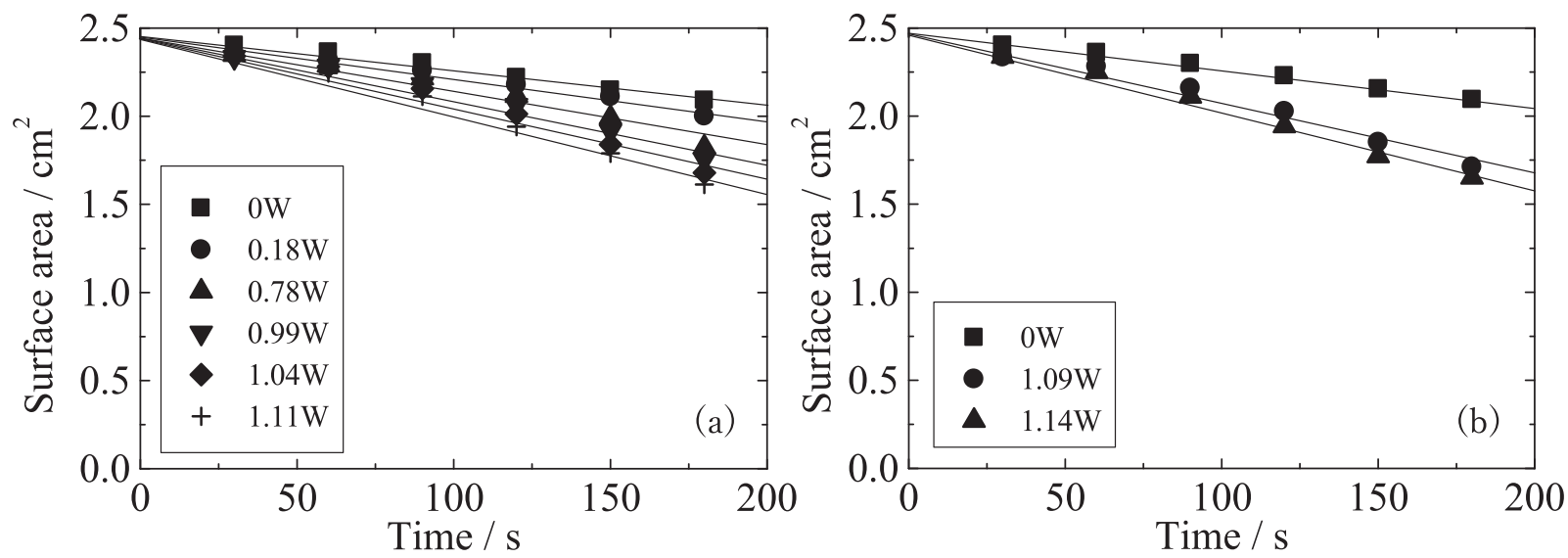

Fig. 8. Comparison of time variation of the surface area of the sample due to differences in the ultrasonic output in the case of $108 \mathrm{kHz}$. (a) non-degassed distilled water (b) degassed distilled water.
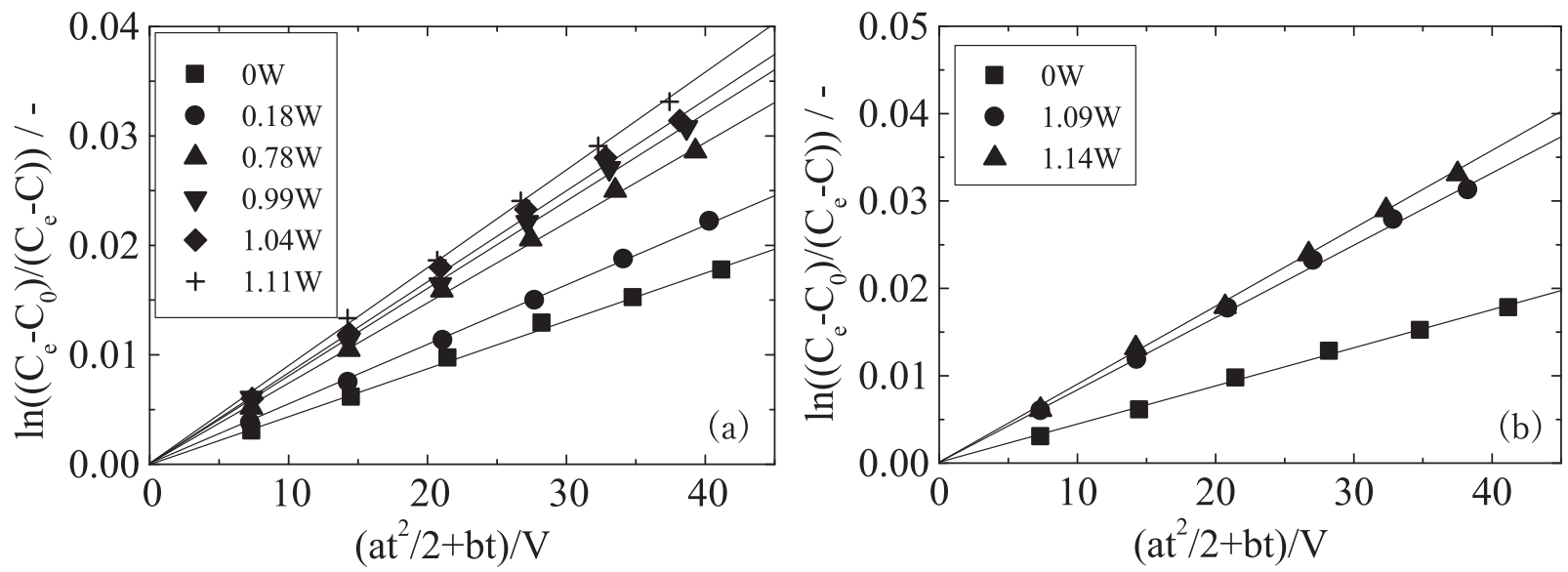

Fig. 9. Analysis by the rate equation of the time-dependent change of sucrose concentration in the case of $108 \mathrm{kHz}$. (a) non-degassed distilled water (b) degassed distilled water.

Figure 10 is a graph showing the effect of ultrasound power on the mass transfer coefficient. Mass transfer coefficient in the degassed water and the non-degassed water had no difference at $108 \mathrm{kHz}$. Although the results of $28 \mathrm{kHz}$ are also plotted in the figure, the mass transfer coefficient was smaller as the frequency was higher. Vibration energy by ultrasound transmitted through the liquid is proportional to the square of the amplitude and frequency. If the ultrasound power input is assumed to be the same between $28 \mathrm{kHz}$ and $108 \mathrm{kHz}$, the amplitude of $108 \mathrm{kHz}$ is smaller than that of $28 \mathrm{kHz}$. Since the ultrasound amplitude is small, the cavitation is less likely to occur. Therefore, the dissolution rate was considered to have slowed. Also in this experiment, the ultrasonic enhancement factor was obtained by the following experimental formula in the case of $108 \mathrm{kHz}$.

$$
E_{W}=\left(1+1.45 P_{W}\right)^{0.66}
$$

\subsection{Effect of Vessel Size on Dissolution Rate}

The experiments with small size vessel were conducted. Ultrasound frequencies were $28 \mathrm{kHz}$ and $108 \mathrm{kHz}$, and nondegassed water was used. Figure 11 shows the relationship between surface area of the sample and time. Linear relationships are obtained in all conditions. Figure 12 shows summarized experimental results by using Eq. (4). As shown in the figure, when ultrasound waves are irradiated into the

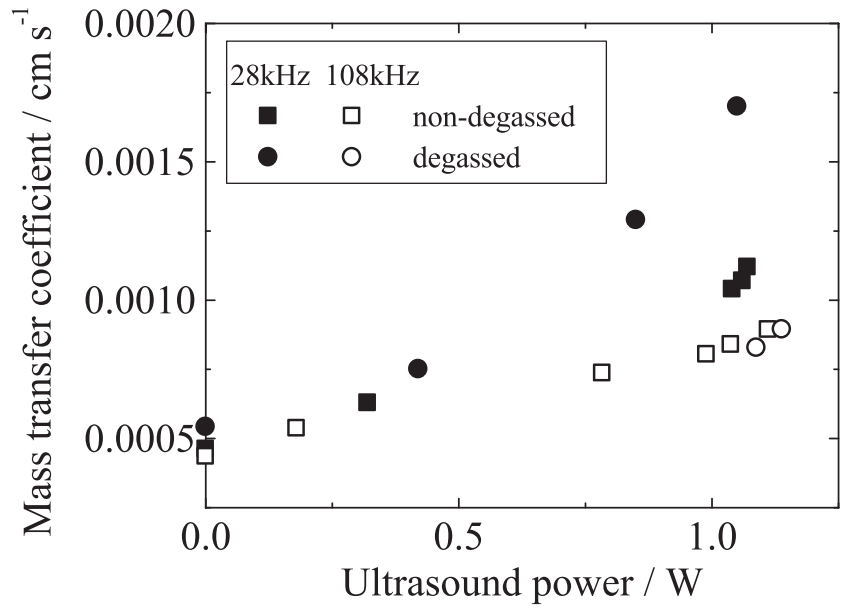

Fig. 10. Influence of ultrasonic power on the mass transfer coefficient.

liquid, the straight lines do not pass through the origin. On the other hand, the linear relations were obtained after $30 \mathrm{~s}$. This indicates that the sample dissolution is fast before $30 \mathrm{~s}$. In the case of using an aluminum container, it is considered that the sound waves irradiated into the liquid was strongly reflected on the wall surface of the container, which caused the cavitation phenomena in the liquid to become active. As non-degassed water was used, it seems that the gaseous 

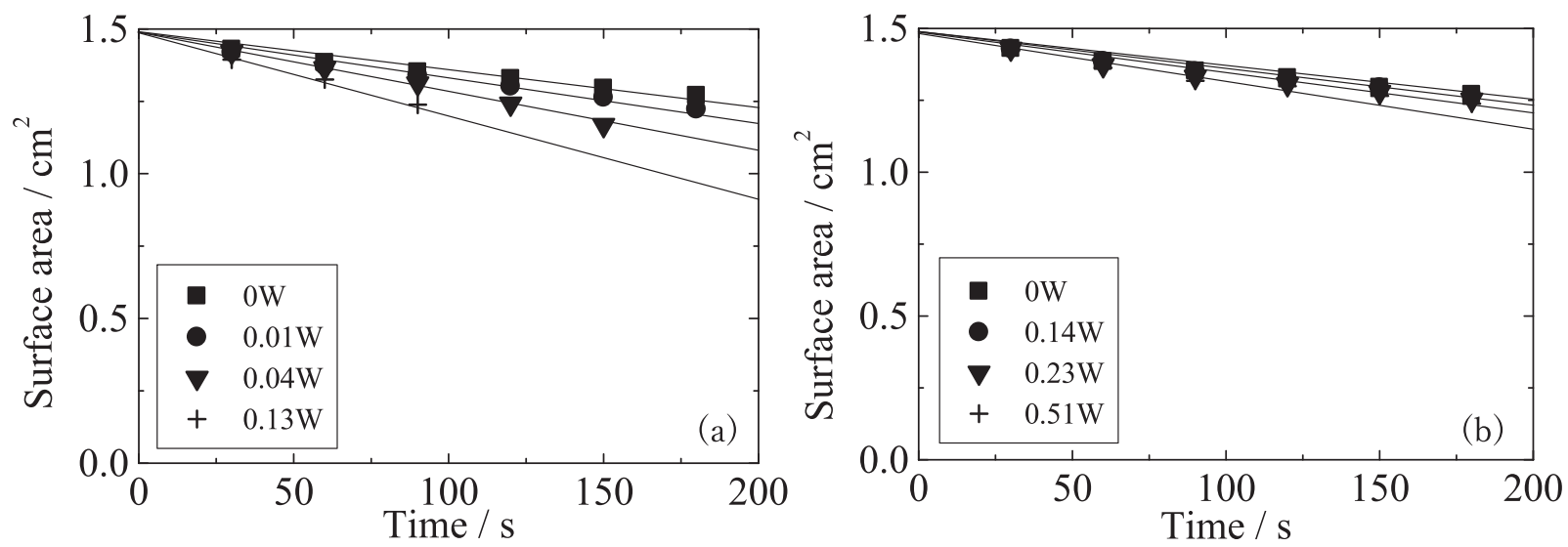

Fig. 11. Comparison of time variation of the surface area of the sample due to differences in the ultrasonic output by using small vessel. (a) $28 \mathrm{kHz}$ (b) $108 \mathrm{kHz}$.
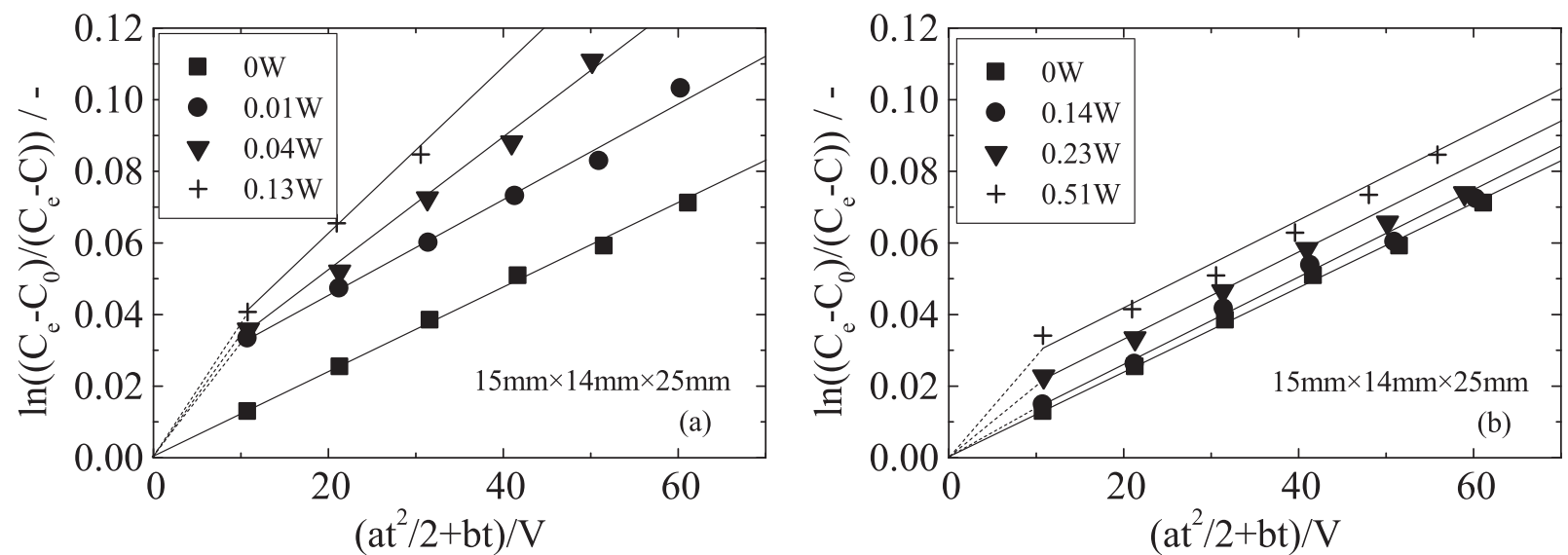

Fig. 12. Analysis by the rate equation of the time-dependent change of sucrose concentration in the case of small vessel. (a) $28 \mathrm{kHz}$ (b) $108 \mathrm{kHz}$.

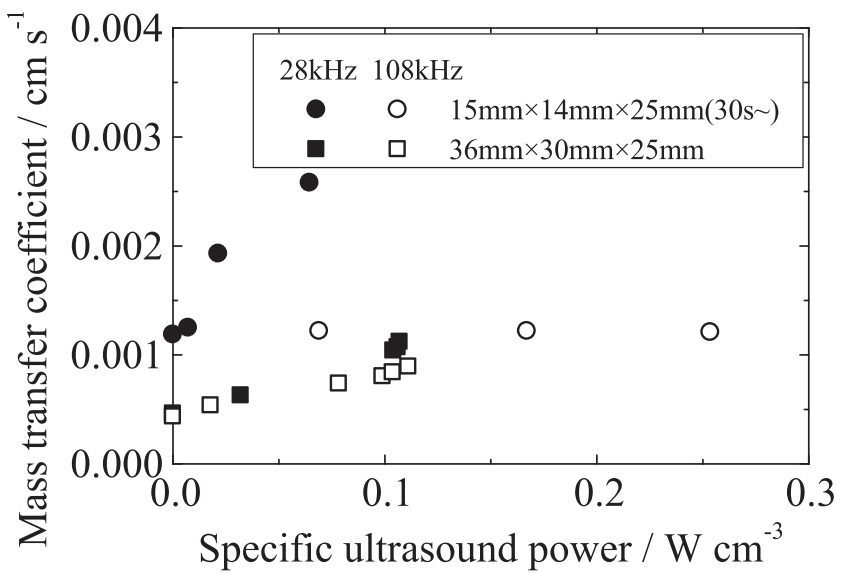

Fig. 13. Influence of specific ultrasound power on the mass transfer coefficient by using non-degassing distilled water.

cavitation became particularly intense before $30 \mathrm{~s}$. After 30 $\mathrm{s}$, the water is degassed. It is thought that the solid dissolution progressed by the vaporous cavitation. Although the influence of ultrasonic power is clear at $28 \mathrm{kHz}$, the effect of ultrasonic power on dissolution rate was not observed at $108 \mathrm{kHz}$. This is considered to be due to the fact that higher frequencies are more difficult to cause the cavitation. Mass transfer coefficient is calculated from the slope of these straight lines. Figure $\mathbf{1 3}$ is a graph showing the effect of

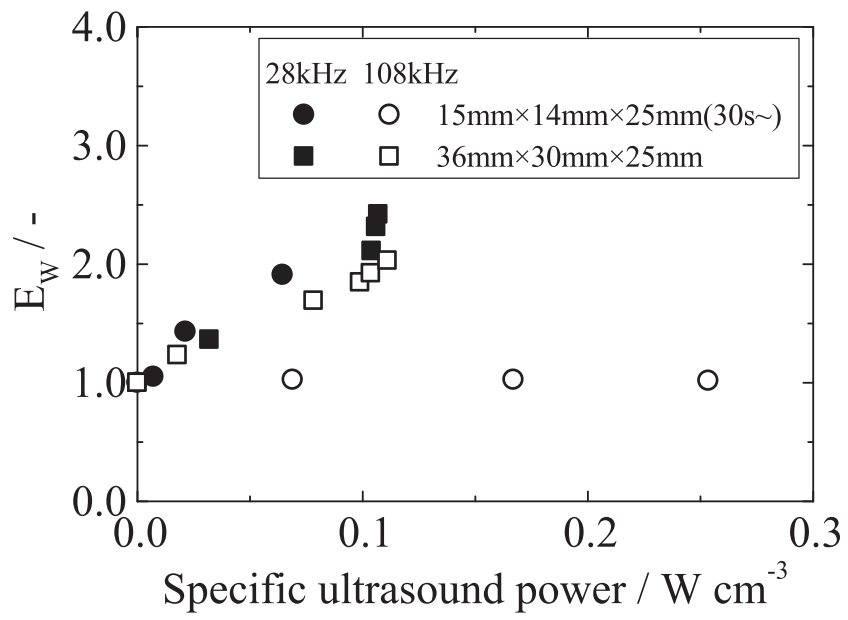

Fig. 14. Influence of specific ultrasound power on the ultrasonic enhancement factor by using non-degassing distilled water.

ultrasound power on the mass transfer coefficient. Considering the vessel size, the horizontal axis of the graph is the ultrasound power per unit water volume. The figure also shows the experimental results of large vessel. The mass transfer coefficient increased for smaller vessels. If the vessel is small, the cavitation occurs near the solid surface, so the dissolution rate of the sample is considered to have been 
promoted. In the case of $108 \mathrm{kHz}$, the mass transfer coefficient did not change. This means that ultrasonic vibration energy is not effectively used for the dissolution acceleration. Figure 14 shows the influence of specific ultrasound power on the ultrasonic enhancement factor, $E_{W}$, by using non-degassing distilled water. The figure also shows the experimental results of large vessel. When the vessel size was large, the enhancement factor became larger at $28 \mathrm{kHz}$ than $108 \mathrm{kHz}$ as the ultrasound power increased. On the other hand, in the case of small vessel, the enhancement factor of $108 \mathrm{kHz}$ was constant even when the ultrasound power increased. In the case of $108 \mathrm{kHz}$, the temperature rising rate of liquid was fast. Therefore, it is considered that the energy of the ultrasound wave was converted to heat without being effectively used for disturbance of liquid near the solid surface. From the above, it is important to activate cavitation in the vicinity of the solid surface at the low ultrasound frequency and disturb the boundary layer of the solid surface to promote dissolution rate of the solid.

\section{Conclusions}

In this study, the enhancement of a solid dissolution rate by applying ultrasound was examined. The effect of ultrasound on the dissolution rate in water of sucrose was investigated. It was found that the following results occurred.

(1) As the ultrasound output becomes large, the dissolution rate is increased.

(2) The sample surface became uneven under the ultrasound irradiation. This phenomenon was considered to be due to the micro-jet at the time of collapse of cavitation.

(3) When degassed water was used, the dissolution rate was more enhanced by the micro-jet at the time of collapse of vaporous cavitation.

(4) When the frequency is large, the cavitation is less likely to occur. Hence, the dissolution rate is not too fast.

(5) In the vicinity of the solid surface the vigorous cavitation phenomena causes on acceleration of the dissolution rate by disturbing the boundary layer.

\section{REFERENCES}

1) S. V. Komarov, M. Kuwabara and O. V. Abramov: ISIJ Int., 45 (2005), 1765.

2) G. I. Eskin: Ultrason. Sonochem., 2 (1995), S137.

3) H. Xu, X. Jian, T. T. Meek and Q. Han: Mater. Lett., 58 (2004), 3669.

4) S. Hatanaka, T. Taki, M. Kuwabara, M. Sano and S. Asai: Jpn. J. Appl. Phys., 38 (1999), 3096.

5) V. Abramov, O. Abramov, V. Bulgakov and F. Sommer: Mater. Lett., 37 (1998), 27.

6) T. Araki: J. Jpn. Inst. Light Met., 11 (1961), 246.

7) M. Matsushima, S. Yadoumaru, K. Mori and Y. Kawai: Tetsu-toHagané, 62 (1976), 182.

8) Y. Yamakado, T. Yamada and K. Iwai: Tetsu-to-Hagané, 102 (2016), 127.

9) A. Said, O. Mattila, S. Eloneva and M. Jarvinen: Chem. Eng. Process., 89 (2015), 1.

10) T. Kamakura, K. Yasuda and Y. Kumamoto: IEICE Trans. Inf. Syst., J80A (1997), 1648.

11) A. Kannan and S. K. Pathan: Chem. Eng. J., 102 (2004), 45.

12) T. Leong, M. Ashokkumar and S. Kentish: Acoust. Aust., 39 (2011), 54.

13) V. Mel'nick, L. Rhuzinska and V. Forostyanko: Technol. Audit Prod. Reserv., 33 (2017), 28.

14) Y. Kato: Cavitation, Maki-shoten, Tokyo, (1999), 204, (in Japanese).

15) H. Grenman, E. Murzina, M. Ronnholm, K. Eranen, J. Mikkola, M. Lahtinen, T. Salmi and D. Y. Murzin: Chem. Eng. Process., 46 (2007), 862.

16) Y. Li, X. Leng, S. Cheng and J. Yan: Mater. Des., 40 (2012), 427.

17) S. Koda: Ultrason. Sonochem., 10 (2003), 149.

18) C. Horst, Y. S. Chen, U. Kunz and U. Hoffmann: Chem. Eng. Sci., 51 (1996), 1837.

19) T. Tekin, D. Tekin and M. Bayramoglu: Ultrason. Sonochem., 8 (2001), 373. 